\title{
Managerial Execution in Public Administration: Practices of Managers When Implementing Strategic Objectives
}

\author{
Alpha Ayande ${ }^{1}$, Vincent Sabourin ${ }^{1} \&$ Ergis Sefa $^{2}$ \\ ${ }^{1}$ School of Management, University of Quebec at Montreal, Canada \\ ${ }^{2}$ ERG Managerial, LLC, Albania \\ Correspondence: Alpha Ayande, School of Management, University of Quebec at Montreal, Canada. Tel: \\ 1-514-661-2400. E-mail: ayande7@bluewin.ch
}

Received: July 4, 2012 Accepted: September 5, 2012 Online Published: October 1, 2012

doi:10.5539/ijbm.v7n19p55 URL: http://dx.doi.org/10.5539/ijbm.v7n19p55

\begin{abstract}
This paper analyzes the dimensions underlying the management practices of public managers when they are implementing their organizational objectives. This study also shows, in relation to our conceptual framework of the five dimensions of strategy execution, the necessary behaviours and attitudes that public managers ought to deploy in performing their missions of public benefit. To that end we conducted a factor analysis, subsequent to a survey of service managers in public administration, which allowed to assess and to emphasize the significance of each of the five dimensions of strategy execution.
\end{abstract}

\section{Methodology}

A survey questionnaire was administered to 367 public managers in the scope of the five dimensions of performance drivers. We use the PCA method to assess the relationship between the dimensions of strategy execution and management practices in public administration regarding the implementation of their organizational objectives.

\section{Findings}

The study found seven main conclusions concerning the sample of public managers surveyed. These findings are:

- The dimension of emotions defined as getting a commitment to the objectives, would be the strongest driver that managers in public administration would use to reach their organizational objectives. The emotions dimension has been supported as the critical dimension.

- The dimension of initiatives defined as translating the objectives into concrete projects, has been found to be the second dimension in order of importance implemented by managers in public services when implementing their objectives.

- The dimension of rules defined as clarifying and aligning the objectives was found to be the third component set out by managers in public administration when executing organizational objectives.

- However, the dimension of integrity (executing the objectives within the integrity of your values) was not revealed as being a critical dimension for managers when implementing the objectives. The dimension of integrity of values was therefore not supported by the study.

\section{Research}

Limitations/Practical implications-the paper reveals the difference in relevance of management practices of public managers in the implementation of their objectives.

\section{Originality and Value}

The study supports the existence of four empirical dimensions in the implementation of managerial objectives in public administration. It brings a new perspective in terms of strategy execution for public service management.

Keywords: public administration, strategy execution, managerial performance, implementation, public management 


\section{Introduction}

Public administration and public management have been changing radically over the past three decades in the United States and across the globe. As business organizations try to adapt to the new globalized environment and market, so do public and government agencies. The most prominent scholar in public administration, Dwight Waldo, defined it as "the organization and management of men and materials to achieve the purpose of public policies set by governments" (Waldo, 1953). Implied in public administration is the crucial role of administrators and managers, who translate into strategies the policies set by governments and then implement them. In the past decade, several authors in public administration and management have put into perspective the need for a better understanding of the topic of strategy execution in organizations (Christensen \& Laegreid, 2008).

For large bureaucracies, like government agencies, strategy execution is almost certainly accompanied with change and transformation (Lane, 2000). That is where problems begin for managers. Change is something that raises barriers and difficulties for public executors (Khan, 2008). According to the Merriam Webster dictionary (2000) the notion of transformation is defined as a change of form and the word transform is defined as a change of structure, appearance or character. Within this definition we find implied the change in practices, the way things are carried out (the change in form), and the internal shift in structures and regulations (change in structure and character). Their impact on the physical, psychological and emotional aspects of employees is obvious. Managers will have to deal with all three of these aspects when trying to execute policies (Lane, 2000).

In this regard, strategy execution is often described in the literature as complex and difficult. Thus, a strategic transformation could be defined as a transformation that is important for the organization. These are the types of strategies managers wish they never had because of the high level of engagement required of them. In this context Lane (2000), argued in a study that 90 percent of the formulated strategies of firms in the USA and Europe are not implemented on time, or with the intended results. Strategic transformations have longer timeframes for the implementation and deliver less anticipated results. Finding the reasons for this lack of performance was not easy. Authors like Berry, (1994) mentioned that often strategy formulation is glamorous but, unfortunately, implementation gets short drifted. Though the great part of the current literature explains the "why", other authors like Christensen \& Laegreid (2008) argued that leaders are now beginning to state that they understand the importance of implementation as well as strategy design. Improvements in the way projects are estimated, scheduled, monitored, and completed have dramatically improved organizational efficiency and effectiveness. As a consequence, these leaders are now asking "What do I need to do differently?" The question most often asked is how a better strategy execution could better execute our strategy to achieve greater organizational performance (Berry, 1994). Especially during the Regan administration and thereafter, even in public agencies, service had been perceived as a category of market offering or as an intangible product offering (Christensen \& Laegreid, 2008). Public managers should turn their agencies into target-achieving machines which maximize efficiency in formal execution but lack long-term commitment by their staff. Public managers are now aware that strategy execution should be accompanied by transformation and change if performance is to be affected. Nevertheless, these transformations and restructurings cannot be fully implemented without a prior deep and comprehensive knowledge of effective service delivery as it relates to the public, whom public agencies are meant to serve.

That is why research on strategy execution in the area of public administration and management practices has highlighted the need to put emphasis on the complexity and the multidimensional aspect of the practices related to it. For instance, Kettler (2002) presented different organizational frames (structural, human resource, political, and symbolic) to better understand and diagnose problems related to implementation. Frant (1993) also argued that this is a combination of several basic elements that match the organizational environment and that explain a successful execution. A classical organizational challenge in complex and large organizations and institutions has been the retaining of the status quo in relation to the changing manager involvement in institutional decision-making (Stivers, 1994). Other authors have argued for a conceptual clarification between managerial skills and broader leadership skills called executive skills to better apprehend strategy execution practices (Ferguson et al., 2010). They argued that often an inquiry into executive skills revolves around the search for managerial skills only and that executive skills form a distinct category of skills to better understand strategy execution. Also others like Bill Damare point to the fact that workplace learning and interacting staff is very important for managers prior and during the implementation of strategies. He argues that this constant interaction with its staff makes a manager more agile in resolving unexpected daily issues which require immediate actions and immediate execution (Damare, 2010). 
In his search for the key elements to better understand management execution practices, Martin (2007) studied factors influencing the human resource management performance of first tier managers, and the vital importance that a holistic strategic framework can have in this regard. Others have investigated the role of management support in a lean implementation (Stivens, Jan/Feb. 2008). For instance, these authors argued that an implementation made on communication within the organization could be simultaneously analyzed using a rational, political and/or intuitive perspective. Others have tried to establish empirical linkages among factors related to strategy execution. For instance Stivers (1994), found that a three-way interaction between change vision clarity, change appropriateness, and change execution was found to predict managers' job satisfaction, turnover intentions, and role ambiguity. Since the last five years, we have seen an intensification of the preoccupation for management practices in strategy execution. Metcalfe (1993) studied the practices of exceptional companies. Zerbe et al. (2010) examined the role of smart HCM. Though the research has recognized the role played by management practices in strategy execution, very few investigations have studied the specific role played by various factors and the relative importance of their dimensions. This is the objective of this paper. A review of the literature in management and of the Kolb model (1984) and subsequent empirical work by Richard and Sabourin (2009); Sabourin (2009); Ayandé and Sabourin (2011) has led us to support the existence of five dimensions adapted to strategy execution in public management. We labeled these dimensions as follows: the dimensions of rules, emotions, initiatives, immediate actions and integrity.

\section{Research Objective}

The objective of this research is to study the dimensions underlying the management practices of public managers when implementing their objectives. The specific objectives of this research are the following:

- Assess empirically the relative importance of the various dimensions in the execution of managerial objectives in public administration.

- Assess the relevance of specific management practices for each of the dimensions of public managers in the execution of their objectives.

\section{Conceptual Framework}

Our conceptual framework is inspired by the work of Kolb (1984) and Kolb and Boyatzis (1995) on experiential learning and additional work on the topic by Richard and Sabourin (2009a); Sabourin (2009a); Ayandé and Sabourin (2011). We found that Kolb's (1984) conceptual model provides us with a complete perspective on the topic of strategy execution. The empirical evaluation of the experiential learning theory was mainly supported in education (Jarvis, 1987; Johnson and coll., 1996; White, 1992). Based on this perspective, our conceptual framework suggests that five different, but complementary dimensions could be factors faced by managers when executing their strategy.

A review of the literature in management and of the Kolb model (1984) and subsequent work (Richard \& Sabourin, 2009; Sabourin, 2009; Ayande \& Sabourin, 2011) has led us to develop a conceptual framework of five dimensions adapted to management leadership in the public sector. We labeled these dimensions as follows:

- The first dimension of rules deals with the clarification and alignment of the manager's objectives. The first dimension gathers variables that refer to factual and rational analyses of given situations. This perspective leads to concept forming and formulation of generalizations that integrate the observations and the reflections. Economic planning and analysis prevail in this dimension. Factors deal with figures, figures and protocols. Decision making is based on facts and abstract principles.

- The second dimension of emotions deals with getting a commitment to the manager's objectives from its employees. This dimension gathers variables dealing with topics such as fetching a commitment, clarifying problems, reconciling the divergent points of view and establishing consensus. In this second situation, we make a thoughtful observation that consists of making observations on the experience lived by the persons and of thinking about their meaning.

- The third dimension of initiatives deals with translating managerial objectives into concrete projects for employees. It gathers variables dealing with the introduction of new projects and ideas that result in more willing and more capable employees. This third dimension relies on the active experimentation of initiatives: realizing projects and continuous improvements to the existing activities.

- The fourth dimension of immediate actions gathers variables that reflect creating value-added actions or immediate actions in response to urgent matters in the execution of objectives. It addresses concrete 
actions and those that allow for rapid actions on a small-scale to obtain quick results. Thus, the variables deal with quick decision taking without respect to an established plan.

- The fifth dimension of integrity deals with executing objectives in the context of the integrity of values and principles. It gathers variables associated with the execution of objectives in respecting organizational values and principles. These variables refer to factors faced concerning organizational values. This is the capacity to realize the organization's objectives in respecting the integrity under pressure.

\section{Review of the Literature}

In the following lines we review the literature according to these 5 dimensions inspired by Kolb (1984).

\subsection{The Dimension of Rules}

The dimension of rules can be defined as a strategy based on a factual analysis of the situations with the intent of setting up a more coherent and more rational system. According to Kolb (1984), this strategy has to do with abstract conceptualization. This strategy leads to the forming of concepts and formulation of generalizations which integrate the observations and the reflections. In fact, the "classical" paradigm of public administration has been considered, ever since Max Weber, to be the only dimension valuable for public management. The view that the administration of public agencies should be dominated by formal regulations was considered to be untouchable. Administrators should implement strategy in a step-down method of strictly following the rules in order to achieve high levels of efficiency (Stivens, Jan/Feb. 2008). In fact, this paradigm was challenged by Dwight Waldo who made possible the shift of this paradigm from public administration to public management. Nevertheless, Waldo emphasized the important role that rules play in the management of an organization, be it public or private. As a result, a new understanding of this dimension has emerged in relation to transformation, change and execution. A first group of authors has examined the management practice of strategy execution as related to what we call the dimension of rules, with factors such as the hierarchy of objective principles of governance. For instance Stivens, Jan/Feb. (2008); Henisz (1999); McLoughlin (1985), studied non-linear governance change by analyzing the importance of sector-specific institutions and the pathways for governing they create. They identified the key role of redistributive policies, more specifically, sector-specific institutions can account for the specific ways in which reforms redefine hierarchy and professional self-regulation and the extent to which reforms strengthen the hierarchy and affect the balance with other forms of governance. McLoughlin (1985); Zerbe et al. (2010) have used the concept of alignment to explain strategy execution and organizational performance.

\subsection{The Dimension of Emotions}

This second dimension of emotions has to do with commitment and the development of employees' convictions in the context of a service transformation. This is what Kolb (1984) labeled a "reflexive observation". It is the most important part of delivering strategy execution as it "clashes" with the internal barriers of resistance to change. These are the most important aspects of resistance to change. A good manager/ administrator will act in order to make its staff/employees become promoters of change, instead of resistance (D'Agostino \& Levine, 2010). This dimension is about how to bring about a commitment from employees by clarifying the problems, reconciling the divergent points of view and establishing consensus. In the context of this strategy, the divergent and conflicting points of view are comfortably accepted. Several authors have underlined the role played by management practices in relation to emotions as a driver of strategy execution. A large number of authors have put emphasis on management practices which could be gathered under the dimension of emotions. In the literature, it has been identified as the predominant perspective on the topic of practices related to strategy execution. Zerbe et al. (2010); McLoughlin (1985) argued that resistance to change represents a key factor in strategy execution and it should also take into account any resistance coming from managers. This resistance is mainly shown as a response to the attack on the interests and power position of the manager. In fact, this has been one of the key problems identified in large public institutions since the beginning of the $21^{\text {st }}$ century. Certainly this was true for the United States, but other countries followed. As Waldo (1953) puts it, administrators tend to routinize their work and their down-the-scale staff adapt to this routine. Executing a new strategy involving change disrupts the established work routine. But the managers themselves will have to change their routines. That is why they become the first barrier to the execution of the strategy. For this reason, a key aspect of execution is the specific support of line managers that must support the change. It has been argued that there should be sufficient leading officials in key positions who completely endorse and extend their cooperation to the changed project (McLoughlin, 1985). 
In their research on the implementation process, McLoughlin (1985); Zerbe et al. (2010) discerned several forces. The most important one is often the force aimed at coordination, which can be traced back to two causes: the idea that implementation must necessarily take place on the job floor; and the idea that many different aspects (components) have to be further developed and coordinated, especially during implementation. Jorgensen (2006) described the processes related to the resistance to implementation in detail. His findings showed how getting a commitment from the resisters is often very complex and difficult. He stated that instead of resisting openly, this group can also manifest itself by taking advantage of every opportunity to postpone any concrete steps towards implementation, in particular by creating procedural delays. In political policy environments (departments, provinces, municipalities), an additional factor is that such delays can also be attempted so that the terms no longer coincide with the realistically required time to complete the implementation.

The research also found support for improved communication in the organization attributable to a lean implementation process. Jorgensen (2006) and Henisz (1999), revealed the psychosocial dimensions of learning as a process that transcends across multiple levels and units of analysis. The analysis of the relationship between individual and organizational learning highlights the multiple and interlocking contexts that define the content and process of learning in organizations, the politics of learning at work and the institutional identity of individuals' learning as a reflection of organizational learning (or lack of it). McLoughlin (1985) examined the relationship between quality of leadership and attitudes, and presented evidence of: the validity of a new leadership instrument; the differential relationship between leadership quality and staff attitudes and their sense of well-being at work; and a predictive relationship between leadership quality and organizational performance. Henisz (1999) explored the linkage between nurses' levels of optimism and performance outcomes. The results indicated a highly significant positive relationship between the nurses' measured state of optimism and their supervisors' ratings of their commitment to the mission of the hospital, a measure of contribution to increase customer satisfaction, and an overall measure of work performance.

All of the above reveal the importance of emotions in the dynamics of an organization, both public and private. Thus, the emotions dimension is the most important aspect of the implementation of a strategy if that strategy is to be successful (Zerbe et al., 2010).

\subsection{The Dimension of Initiatives}

The dimension of initiatives relies on the active experimentation of initiatives, the realization of projects and continuous improvements to the existing activities. This is what Kolb (1984) called the process of active experimentation. Extensive literature shows the significance of the learning function in the organization based on innovation and projects as a way to execute organizational objectives (Rainey, et al., 1976; Park \& Rainey, 2008; Berry, 1994). Many practitioners and academics now endorse the view that strategy execution is based on decentralized initiatives and small-scale improvements and individual innovations that help to attain organizational success (Metcalfe, 1993; Berry, 1994; Ring, \& Perry, 1985; Van der wal, et al., 2006). Taking initiatives during the daily routine of work proved to be a must, especially for field managers. One perfect example of such a situation is the study conducted by Karakosta, Doukas and Psarras (2009) regarding a development project conducted in Chili and sponsored by the World Bank. They demonstrated the importance of taking initiatives from field managers. They argue that without the constant initiatives and immediate actions of these down-the-scale managers, the level of performance of their workers would be significantly lower. That would directly impact the overall efficiency of the organization and thus the realization of the strategy execution process.

Other authors like Berry (1994), have shown how an inventory of leader behaviours likely to enhance employees' innovative behaviour, and the initiatives and projects of employees are a factor in the execution of objectives. According to Van der wal, et al. (2006), the manager function is more explicit and takes on a more general responsibility for the department and the professions. However, the managerial function is substantiated by conditions related to the professional field of knowledge, which gives legitimacy within a medical logic. Managerial changes need to relate to this if they are to have consequences on the managerial roles and structures at the departmental level in hospitals. The paper suggests that the future development of this role will depend on the way the collectivist and individualist aspects of responsibility are handled, as well as on the further development of the managerial knowledge of physicians. Some authors have examined the role of decentralization and initiatives as management and organizational practices in the execution of objectives. For instance Warwick (1975) when discussing recent research contributions in comparative healthcare systems, emphasized decentralization as a major characteristic of Scandinavian hospital systems. 
In fact, the lack of initiatives from managers and administrators is something that even Waldo warned against more than six decades ago (Waldo, 1953). This lack of initiatives was identified by him as one of the major factors influencing the lack of efficiency in large bureaucracies. Second only to this was the lack of immediate actions on issues that come up during the daily routine.

\subsection{The Dimension of Immediate Actions}

Fourthly, the dimension of immediate actions is carried out through immediate actions and allows for an immediate implementation at a small-scale level in order to obtain results quickly and adjust on the basis of feedback. According to Kolb (1984), results are obtained by means of immediate actions. There is an opportunity for fast decision-making without respect to an established plan. Interaction with the others is favoured only as far as it gives results quickly. This perspective is partially inspired by the theory of the contingency school (Ferguson, et al., 2010), according to which the execution depends widely on the immediate context. This perspective meets itself, however, mainly in works intended for the practitioners and often outside of university research. These works present a voluntarist perspective rather than a determinist one. Under this perspective, the success of the execution rests on the leadership of the managers (Jolliffe, 2002; Khan, 2008) and the capacity to take action in a concrete and immediate way in difficult situations. Warwick (1975) with his various books is a good representative of the perspective. Covey emphasizes the problems of the urgent matter as a symptomatic element of the lack of control of the administrator in the execution of organizational objectives. For Lane (2000), the immediate actions and the implementation of actions are the elements-keys in the execution of a strategy. Damare (2010) in his study of CEOs found even at this level that more than half of their activities lasted less than nine minutes. Jolliffe (2002) painted a picture of how a lack of time stresses a person who is constantly interrupted and unable to prioritize, and therefore has no time for longer-term planning. Berry (1994) and Ferguson et al. (2010) identified short-term work pressures as an obstacle to strategy execution. Altshuler and Behn (1997) studied the prioritization process to derive a set of factors dealing with too many conflicting priorities in terms of customer needs, capability risks, directives, initiatives, issues and activities. Management engineers are the hostages of short-term immediate actions and recurrent short emergencies that impede long-term efforts. Damare, et al. (2010)) refer to the practice as a unified approach to development versus a piecemeal one. They argue that a unified approach rather than one regarding development as a separate and isolated process is a key practice for HRD managers. Their argumentation is also based on the work of Jolliffe (2002), who argues that when his managerial development is not linked to a business strategy and when activities are unrelated and fail to reinforce each other, they reduce the potential for improving organizational effectiveness. In this regard, Henisz (1999) showed the benefit of a prioritization process developed and used at Network system of BAE Systems. This system has allowed for better immediate actions and facilitated trade-offs in prioritizing goals, customer needs, capabilities and technical performance with an improved prioritization process. This strategy is pragmatic and acts on the basis of the first results obtained. For instance, Jolliffe (2002) mentioned that a relevant topic is the assessment of reactions to organizational change.

\subsection{The Dimension of Integrity of Values}

The dimension of integrity deals with the capacity to achieve objectives within the values and principles of the organization. Executing objectives in the context of integrity has to do with the capacity to achieve objectives without compromising the values and principles in the functioning of the organization. Several authors have put emphasis on the integrity of values and principles as a way to execute objectives. For instance Frant (1993), mentions that one of the forces in implementation is the organizational force to redefine the identity. Henisz (1999) identified transparency, for instance, as a key value linked to strategy execution and organizational performance as it highlights the role of a superordinate identity. For achieving team goals in an organization, Kettl (2002) and Fernandez and Rainey (2006) suggest that project leaders have a role in developing team characteristics into a collective set of virtues including: Ethics, Respect and Trust for others, Honesty, Prudence, Courage, Responsible Use and Sharing of Power. Many managers tend to form a framework of values during their daily routine with their staff. Many authors call this framework of values the "work culture", or "organizational culture". This organizational culture represents the boundaries within which strategy should be executed. The problem with these "boundaries" is that in time they turn out to be barriers to growth or innovation as new conditions appear (Karakosta et al., 2009). Nevertheless, in many cases they turn out to be "positive barriers" against corruption and abuse.

In conclusion, according to the review of the literature, the management practices for executing the objectives of the public managers should be structured around the following hypotheses. 


\section{Hypotheses}

In accordance with the review of the literature and our conceptual framework, we could formulate the following six hypotheses.

Main hypothesis

$\mathbf{H}_{1}$ : The management practices related to the dimension of emotions such as the capacity to level-down resistance to change and to meet commitments would be used more intensively by managers than any other dimension of execution when executing their objectives.

Our hypothesis is that managers use this dimension extensively in order to increase the collaboration among employees and reconcile divergent points of view, which can be a major obstacle to strategy execution. A key aspect of strategy execution is the support of line managers that deal with daily resistance from the established company work culture. That is why managers, from the top down to the bottom line, extensively use the emotions dimension to "soften" this resistance when implementing new strategies.

According to the review of the literature and our conceptual framework, we could also formulate the following five specific hypotheses.

Secondary hypotheses

$\mathbf{H}_{2}$ : The management practices that relate to the dimension of emotions such as the capacity to develop convictions and to get a commitment represent a significant component of practices for managers when executing their objectives.

This secondary hypothesis is related to the ability of managers to level-down resistance to change when implementing a strategy. A change in organizational culture begins with the conviction that something has to be changed. It continues with the commitment of the down-the-line managers and employees who will work toward the implementation of this change.

$\mathbf{H}_{3}$ : The management practices that relate to the dimension of taking initiatives such as learning by doing and giving the employees a sense of responsibility are a significant component of practices for managers when executing their objectives.

In order to spearhead change in the organizations, managers are obliged to take advantage of windows of opportunities and to undertake initiatives and actions without any prior planning. This will set an example for the other line managers and employees and will also give them a sense of responsibility in the process of implementation of the strategy.

$\mathbf{H}_{4}$ : The management practices that relate to the dimension of rules such as clarifying and aligning the objectives, are a significant component of practices for managers when executing their objectives.

Even though initiatives are important to trigger the process of implementation of a strategy, nevertheless, in order to have an effective implementation, managers need to clarify their protocols and objectives. This is accomplished in order to avoid confusion among employees about the specific tasks expected from them. Sometimes this includes even the rules and procedures of executing a task in order to yield the maximum efficiency.

$\mathbf{H}_{5}$ : The management practices that relate to the dimension of immediate actions, such as quick feedback and direct contacts, represent a significant component of practices for managers when executing their objectives.

As described in the third hypothesis above, managers take advantage of windows of opportunity to take actions to ensure the proper implementation of a strategy. Direct contact with down-the-line employees can serve as a source of feedback for possible strategy modifications and as a morale booster for them. Managers try to take advantage from these opportunities to support execution.

$\mathbf{H}_{6}$ : The management practices that relate to the dimension of integrity, such as practices related to the clarification of values, represent a significant component of practices for managers when executing their objectives.

When implementing objectives, managers are careful not to cross the borders of the guiding values and principles of the organization. Such a trespass could result in long-term negative consequences for the organization. That is why when laying down the strategy implementation rules, managers are eager to clarify the limits within which any action should be undertaken. 


\section{Methodology}

The research is part of a broader research on managerial strategy execution and was conducted in four major steps.

Step 1: Empirical validation of the dimensions of our conceptual framework

1) Data was collected by managers through structured training in countries members of the Organization for Economic Co-operation and Development (OECD). Three regions of the world, namely, Europe, North America and Australia, were selected. 322 referees completed the questionnaire.

2) The measuring instrument of Kolb and Kolb (2005), which is the 'Learning Style Inventory', has been used ever since the initial variables were related to the modes of learning. We validated the questions during executive seminars with the managers of the organization.

3) To make sure that each of the questions was understood, the validation was preceded by a pre-test conducted on 15 referees of the Belgian Management Training Association. All questions were suitably understood and adjustments were made to one in order to clarify its understanding.

4) Descriptive analyses were completed to identify certain characteristics of the sample. A Frequency analysis and the test of Cronbach Alpha were completed. The results of R-square (degree of explained variance by the model) and factorial analyses were used to verify the hypotheses. As shown in Table 2, reference is made to the Cronbach Alpha-an indicator of reliability with the scale of measure between 1 (not reliable of the whole) and 5 (reliable).

5) Four of the five dimensions of our conceptual framework have been validated in previous research. The first four dimensions had a positive Cronbach alpha (Table 1) and the fifth dimension was added afterwards following meetings with the focus groups. Table 1 below presents the concept definition and the variance and reliability obtained in previous work (Richard \& Sabourin 2009).

Table 1. Concept definition

\begin{tabular}{|c|c|}
\hline Concept definition & Variance and reliability \\
\hline $\begin{array}{l}\text { Rules(abstract conceptualization): theoretical conceptualization by means of } \\
\text { rules, postulates and models to systematize information }\end{array}$ & $\begin{array}{l}\text { Variance explained: } 53.5 \% \\
\text { Alpha of Cronbach: } 0.799\end{array}$ \\
\hline $\begin{array}{l}\text { Emotions (reflexive observation): problem recognition and capacity to develop } \\
\text { convictions and to get a commitment }\end{array}$ & $\begin{array}{l}\text { Variance explained: } 60 \% \\
\text { Alpha of Cronbach: } 0.831\end{array}$ \\
\hline $\begin{array}{l}\text { Initiatives (active experimentation): select a model to test its possible } \\
\text { consequences. Learning by trying, finding new ways to put new ideas into } \\
\text { practice. Support initiatives to responsibilize employees. }\end{array}$ & $\begin{array}{l}\text { Variance explained: } 53 \% \\
\text { Alpha of Cronbach: } 0.8\end{array}$ \\
\hline $\begin{array}{c}\text { Immediate actions: oriented actions that are immediate and concrete. Oriented } \\
\text { towards direct contacts and apprehension rather than comprehension. Quick } \\
\text { adjustments resulting from feedback. }\end{array}$ & $\begin{array}{l}\text { Variance explained: } 52.6 \% \\
\text { Alpha of Cronbach: } 0.740\end{array}$ \\
\hline $\begin{array}{l}\text { Integrity: ability to meet organizational objectives in respecting the integrity of } \\
\text { the mission. Active and deliberate construction of the organization's values into } \\
\text { the structure of its everyday actions. }\end{array}$ & Not applicable \\
\hline
\end{tabular}

Step 2: Focus groups to identify managerial factors

Twelve (12) focus groups were conducted with an average of 15 managers per group to identify factors faced by managers. The drivers identified were used as input to elaborate the measurement instrument related to the drivers. 
Table 2. Description of measurement questions in the dimensions

\begin{tabular}{|c|c|c|}
\hline Drivers & $\begin{array}{l}\text { Dimensions and } \\
\text { variables }\end{array}$ & Measurement Questions \\
\hline & Dimension of & \\
\hline & Rules & \\
\hline $\operatorname{Drv}_{1}$ & $\mathrm{~V}_{1}$ & I have some techniques to clarify the expectations of our managers. \\
\hline $\operatorname{Drv}_{2}$ & $\mathrm{~V}_{2}$ & $\begin{array}{l}\text { We evaluate each year our service and the goals oriented toward the clients' } \\
\text { relationship satisfaction. }\end{array}$ \\
\hline $\operatorname{Drv}_{3}$ & $\mathrm{~V}_{3}$ & $\begin{array}{l}\text { We have techniques so that our people are results-oriented with respect to our } \\
\text { annual objectives. }\end{array}$ \\
\hline $\operatorname{Drv}_{4}$ & $\mathrm{~V}_{4}$ & $\begin{array}{l}\text { We systematically conduct annual reviews of our activities with the other } \\
\text { units within our organization. }\end{array}$ \\
\hline \multirow[t]{3}{*}{$\operatorname{Drv}_{5}$} & $\mathrm{~V}_{5}$ & $\begin{array}{l}\text { We are able to estimate the economic value of improvements we wish to } \\
\text { make throughout the organization. }\end{array}$ \\
\hline & Dimension of & \\
\hline & Emotions & \\
\hline $\operatorname{Drv}_{6}$ & $\mathrm{~V}_{6}$ & $\begin{array}{l}\text { We are able to encourage our workers to adhere to our goals so that they are } \\
\text { fully aware of their importance. }\end{array}$ \\
\hline $\operatorname{Drv}_{7}$ & $\mathrm{~V}_{7}$ & $\begin{array}{l}\text { We are able to communicate a sense of urgency to our workers so that they } \\
\text { are able to make rapid decisions. }\end{array}$ \\
\hline $\operatorname{Drv}_{8}$ & $\mathrm{~V}_{8}$ & $\begin{array}{l}\text { We are able to significantly increase the motivation and levels of engagement } \\
\text { of our workers. }\end{array}$ \\
\hline $\operatorname{Drv}_{9}$ & $\mathrm{~V}_{9}$ & $\begin{array}{l}\text { We work closely with colleagues who are able to support us during the } \\
\text { decision-making process. }\end{array}$ \\
\hline \multirow[t]{2}{*}{$\operatorname{Drv}_{10}$} & $\mathrm{~V}_{10}$ & We are able to treat our employees fairly. \\
\hline & $\begin{array}{l}\text { Dimension of } \\
\text { Initiatives }\end{array}$ & \\
\hline $\operatorname{Drv}_{11}$ & $\mathrm{~V}_{11}$ & We have developed a culture that fosters initiative and accountability. \\
\hline $\operatorname{Drv}_{12}$ & $\mathrm{~V}_{12}$ & We translate our goals into concrete projects for all our employees. \\
\hline $\operatorname{Drv}_{13}$ & $\mathrm{~V}_{13}$ & We are able to develop team-based objectives. \\
\hline $\operatorname{Drv}_{14}$ & $\mathrm{~V}_{14}$ & $\begin{array}{l}\text { In my organization, we use various techniques of self-resolution at the } \\
\text { employee level in order to solve the management dilemmas they meet. }\end{array}$ \\
\hline $\operatorname{Drv}_{15}$ & $\mathrm{~V}_{15}$ & $\begin{array}{l}\text { In my organization, we use various techniques according to the level of } \\
\text { importance of the decisions and team-based management. }\end{array}$ \\
\hline
\end{tabular}




\begin{tabular}{|c|c|c|}
\hline & $\begin{array}{l}\text { Dimension of } \\
\text { Immediate } \\
\text { Actions }\end{array}$ & \\
\hline $\operatorname{Drv}_{16}$ & $\mathrm{~V}_{16}$ & $\begin{array}{l}\text { We systematically provide improvements and contingency plans to } \\
\text { effectively respond to emergencies. }\end{array}$ \\
\hline $\operatorname{Drv}_{17}$ & $\mathrm{~V}_{17}$ & $\begin{array}{l}\text { Over the past few years, the number of emergencies we responded to has } \\
\text { decreased. }\end{array}$ \\
\hline $\operatorname{Drv}_{18}$ & $\mathrm{~V}_{18}$ & $\begin{array}{l}\text { We systematically perform reviews to find sustainable solutions for repeat } \\
\text { situations. }\end{array}$ \\
\hline $\operatorname{Drv}_{19}$ & $\mathrm{~V}_{19}$ & $\begin{array}{l}\text { I dedicate at least } 2 \text { to } 3 \text { ninety-minute sessions each week to work directly on } \\
\text { their annual goals. }\end{array}$ \\
\hline \multirow[t]{2}{*}{$\operatorname{Drv}_{20}$} & $\mathrm{~V}_{20}$ & We dedicate a maximum of one day each week to respond to urgent requests. \\
\hline & $\begin{array}{l}\text { Dimension of } \\
\text { Integrity }\end{array}$ & \\
\hline $\operatorname{Drv}_{21}$ & $\mathrm{~V}_{21}$ & We clearly define the values of our organization. \\
\hline $\operatorname{Drv}_{22}$ & $\mathrm{~V}_{22}$ & When under pressure, we are able to reinforce the values of our organization. \\
\hline $\operatorname{Drv}_{23}$ & $\mathrm{~V}_{23}$ & $\begin{array}{l}\text { I am able to recognize differences between the values of my employees and } \\
\text { those of my organization. }\end{array}$ \\
\hline $\operatorname{Drv}_{24}$ & $\mathrm{~V}_{24}$ & We are reputed for the quality of our service delivery. \\
\hline $\operatorname{Drv}_{25}$ & $\mathrm{~V}_{25}$ & We have some techniques to reinforce our employees' sense of responsibility. \\
\hline
\end{tabular}

Step 3: Development of a measurement instrument

We further developed an instrument tool to measure the role of the 25 drivers that were identified by the focus groups. The questionnaire was administered and the questions were sequentially adjusted with five groups of approximately 25 managers per group.

Step 4: Surveys of managers

Once the instrument was validated, we surveyed 367 managers to better understand the drivers they deploy in the execution of their objectives. The objectives are to empirically assess the existence of the 5 categories of drivers. The number of variables was reduced from 25 to 19 after examination of the empirical findings for the section on integrity. Table 3 below presents the dimensions and the variables.

Step 5: Sample for the survey of managers

In the specific context of this research we surveyed a specific group of 367 managers in order to better understand the factors facing them when executing their objectives. In our sample, an average of $48.2 \%$ of the managers was responsible for 5 to 19 employees working under them. The median years of service at the current organization was 2 to 5 years of which a majority $(36.2 \%)$ had spent less than 2 years at their current managerial position. A majority of the respondents $(60.5 \%)$ were 49 years old or younger. On average $34.1 \%$ of the managers had been employed by the organization for a time-period lasting between 2 and 10 years. The median years of service at the current organization was 10 to 20 years of which a majority (68.5\%) had spent less than 20 years at their current organization.

Step 6: Identification of the 5 dimensions with Principal Component Analysis for the specific purpose of this study

Once the instrument was validated, we surveyed 406 managers to better understand which of the performance drivers they used to implement the execution of their objectives. The objectives empirically assess the existence 
of 5 categories of performance drivers. The number of factors was reduced from 25 to 15 after examination of the empirical findings for the section on integrity. Table 3 presents the dimensions and the variables.

It was found that some variables at the end of the matrix rotation had migrated to others dimensions. Therefore, these variables were correlated to other dimensions of the concept model. Also, 6 of the 25 variables weren't correlated and were extricated from the factor analysis. This change in relation to the primary configuration of the four dimensions is remarkable, given that many variables that had formerly belonged to one particular dimension were incorporated in other dimensions. Ultimately, 19 instead of 25 variables have been linked and taken into account in the study.

Table 3. KMO and Bartlett's Test

Kaiser-Meyer-Olkin Measure of Sampling Adequacy 0.926

\begin{tabular}{lll}
\hline Bartlett's Test of Sphericity $\quad$ Approx. Chi-Square & 4572.037
\end{tabular}

Df

Sig.
300

0.000

\section{Data Analysis}

\subsection{The Drivers of the Rules Dimension (Means $V_{1}$ to $V_{5}$ )}

According to our conceptual framework, the first dimension of rules deals with the clarification and alignment of the managers' objectives. The first dimension gathers variables that refer to a factual and rational analysis of the situations that arise from the implementation of a strategy or during the managers' daily work. Below is a description of what the data gathered from the survey regarding each of the variables of this dimension.

$\mathbf{V}_{1}$ : I have some techniques to clarify the expectations of our managers. Overall, $91.8 \%$ of the respondents are clear about the results he/she is expected to achieve with their managers and the organization. Only $8.2 \%$ of them believe that in their organization there is lack of clarity regarding the results they are expected to achieve (median score 2.44, standard deviation 0.984).

$V_{2}$ : Each year we evaluate our service and goals which are oriented toward the clients' satisfaction. For our second variable in the dimensions of rules, the emphasis is placed on clients' satisfaction. An overall majority of $71.8 \%$ indicated in the affirmative that the emphasis placed on customer service exceeded that placed on financial and compliance rules (median score 2.33, standard deviation 1.188).

$V_{3}$ : We have techniques to ensure that our people are results-oriented regarding our annual objectives. When questioned about the lack of orientation in the results and objectives to be achieved, overall, $85 \%$ of the managers think that they display a clear understanding of the results that their respective managers need to achieve (median score 2.55, standard deviation 1.001).

$\mathrm{V}_{4}$ : We systematically conduct annual reviews of our activities with the other units of our organization. More than $64.1 \%$ of the respondent managers state that annual reviews were held with other work units. Only $12.7 \%$ of them agree there is a lack of communication with other departments (median score 2.85, standard deviation 1.338).

$V_{5}$ : We are able to estimate the economic value of the improvements we wish to make throughout the organization. $63.6 \%$ of the respondent managers state that they were able to estimate the economic value of the desired improvements to their organizations. Less than $13.9 \%$ of them agree there is a lack of communication with other departments (median score 3.11, standard deviation 1.177).

\subsubsection{Obstacles to the Dimension of Rules (Means V1 to V5)}

From the mean score of the 5 variables above we clearly see that $57.52 \%$ of the managers agree that the presence of a rules dimension, which deals with the clarification and alignment of the manager's objectives, is necessary and clear to them. Even less than $10 \%$ of these managers (taking 2.59 as middle point) think that a lack of clarification and alignment of the manager's objectives with that of the organization and their staff is an obstacle. 


\subsection{The Dimension of Emotions}

According to our conceptual framework, the second dimension of emotions deals with getting a commitment to the manager's objectives from its employees. This dimension gathers variables dealing with topics such as getting a commitment, clarifying problems, reconciling the divergent points of view and establishing a consensus.

$V_{6}:$ We are able to encourage our workers to adhere to our goals so that they are fully aware of their importance. With the indicated values, the average median score is 2.79 and standard deviation 0.931 . Nearly $84.5 \%$ of the managers understand how to encourage their workers so that they fully adhere to the importance of the organization's goals. Only $3.2 \%$ of the managers think there are no methods to gain the support of their objectives by the employees.

$V_{7}$ : We are able to communicate a sense of urgency to our workers so that they are able to make rapid decisions. A significant majority $(86.3 \%)$ of the surveyed managers were able to communicate in situations of emergency and make rapid decisions. On the contrary, only $3.2 \%$ of the managers were not able to communicate when it was urgent to make a decision (median score 2.64, standard deviation 1.000).

$V_{8}$ : We are able to significantly increase the motivation and levels of engagement of our workers. More than $77 \%$ of the managers think that they have sufficiently motivated their employees to increase their level of engagement. $4.7 \%$ of the managers were unable to increase the engagement level of their employees (median score 2.93, standard deviation 0.897 ).

$V_{9}$ : We work closely with colleagues who are able to support us during the decision-making process. $80 \%$ agree with the fact that they need to work with competent colleagues and to count on their support, while $3.2 \%$ consider that they do not correspond to their organizational practices (median score 2.72, standard deviation 1.023).

$V_{10}$ : We are able to treat our employees fairly. $86.7 \%$ of the managers treat their employees fairly, whereas $4.4 \%$ of them don't. We also note a median score of 2.48 and a standard deviation of 1.050.

\subsubsection{Obstacles to the Dimension of Emotions (Means $\mathrm{V}_{6}$ to $\mathrm{V}_{10}$ )}

Overall, in calculating the mean frequency and score for the variables under the dimension of emotions, a majority of $88 \%$ (taking 2.795 as the mid-point score, $100 \%-11.7 \%=88.3 \%$ ) of the respondents do not evaluate the emotions dimension as an obstacle to their ability to perform the managerial tasks assigned to them. This reflects that the managers are able to get a commitment from their respective employees to fulfill the managers' objectives.

\subsection{The Dimension of Initiatives}

According to our conceptual framework, the third dimension of initiatives deals with translating managerial objectives into concrete projects for employees. It gathers variables dealing with the launching of new projects and ideas that result in more willing and more capable employees. This third dimension relies on the active experimentation of initiatives: realize projects and continuous improvements of the existing activities.

$V_{11}$ : We have developed a culture that fosters initiative and accountability. $79 \%$ of the managers had implemented a culture of initiative and accountability while $4.5 \%$ had not. For this variable the median score is 2.78 and the standard deviation is 1.089 .

$\mathbf{V}_{12}$ : We translate our goals into concrete projects for all our employees. On average, $75.2 \%$ of the managers state that their employees are concerned with their organization's goals. Only $4.8 \%$ of the managers translating goals into concrete projects for all employees were unconcerned by their aim. It has a median score of 2.89 and a standard deviation of 1.054 .

$\mathbf{V}_{13}$ : We are able to develop team-based objectives. This concerns more than $78 \%$ of the managers while $3.8 \%$ could not develop techniques to orient teams to their objectives (median score 2.77, standard deviation $1.034)$.

$V_{14}$ : In my organization, we use various techniques of self-resolution at the employee level in order to solve the management dilemmas they meet. $65.4 \%$ of the managers agree that they used these various techniques while $10.9 \%$ of the managers surveyed did not translate this aspect into practice. For this observation, the median score is 3.07 and the standard deviation is 1.138 .

$V_{15}$ : In my organization, we use various techniques according to the level of importance of the decisions and team-based management. $73.1 \%$ of the managers considered that they used these various techniques 
relative to the decision's level of importance and team-based management, and $7.1 \%$ of the managers surveyed did not translate this aspect into practice (median score 2.94, standard deviation 1.068).

\subsubsection{Obstacle to the Initiatives Dimension (Means V11 to V15)}

Observing the mean score for variables 11 to 15 dealing with the dimension of initiatives, the authors found that with a median score of 3.5 and standard deviation of $0.86,84 \%$ (taking 2.92 as mid-point, $100 \%-15 \%=9 \%$ ) of the test sample respondents feel that the dimension of initiatives was not an obstacle to translate managerial objectives into concrete projects for employees.

\subsection{The Dimension of Immediate Actions}

According to our conceptual framework, the fourth dimension of immediate actions gathers variables that reflect creating value-added actions or immediate actions in response to urgent matters in executing the objectives.

$V_{16}$ : We systematically provide improvements and contingency plans to effectively respond to emergencies. With a median score of 3.00 and a standard deviation of $1.067,73.2 \%$ of the respondents agree that they systematically provide improvements and contingency plans in order to effectively solve emergency situations. Overall, $5.4 \%$ of them disagree, stating that this variable was a restraint to the performance of their managerial duties.

$V_{17}$ : Over the past years, the number of emergencies we responded to has decreased. When asked about the number of emergencies tackled, the median score was of 3.43 (standard deviation 1.191). The managers who agreed represent $47.9 \%$ of the sample.

$\mathrm{V}_{18}$ : We systematically perform reviews to find sustainable solutions for repeat situations.

Most managers agree and they represent on average $89 \%$ of the respondents (median score 3.03, standard deviation 1.163). $10.9 \%$ of them consider that there is a lack of performing reviews to find sustainable solutions.

$V_{19}$ : I dedicate at least 2 to 3 ninety-minute sessions each week to work directly on their annual goals. Only $41.2 \%$ of the managers dedicated a time to work on the achievements of their organization's annual goals. This variable screens a median of 4.00 and a standard deviation of 1.233.

$\mathbf{V}_{20}$ : We dedicate a maximum of one day each week to respond to urgent requests. With a median score of 2.98 and a standard deviation of $1.343,14.1 \%$ of the respondents disagree, saying they don't have the available time to respond to urgent requests; on the contrary, $81 \%$ of managers agreed.

7.4.1 Obstacles to the Dimension of Immediate Actions (Means V16 to V20)

Calculating the mean score frequency for variables 16 to 20 that deal with the dimension of immediate actions, the authors found that with a median score of 3.2 (standard deviation 0.9 ), $56.7 \%$ of the total respondents indicate that the variables included in the dimension of immediate actions (v16 to v20) pose an obstacle to their managerial objectives.

\subsection{The Dimension of Integrity}

According to our conceptual framework, the fifth dimension of integrity deals with executing objectives in the context of integrity. It gathers variables dealing with executing objectives while respecting organizational values and principles.

$\mathbf{V}_{21}$ : We clearly define the values of our organization. With an average median score of 2.52 (standard deviation 1.055), variable 21 indicates that overall, $83 \%$ of respondents feel that there are shared organizational values.

$\mathbf{V}_{22}$ : When under pressure, we are able to reinforce the values of our organization. This variable screens a 2.84 median and a 0.958 standard deviation. More than $76.1 \%$ of managers feel there is no gap between personal and organizational values.

$V_{23}$ : I am able to recognize differences between the values of my employees and those of my organization. Overall, $83.9 \%$ of managers focus on how to recognize these differences between personal organizational values, with a median average score of 2.62 (standard deviation 0.939 ).

$\mathbf{V}_{24}$ : We are reputed for the quality of our service delivery. More than $72.3 \%$ of managers agree with their reputation of quality service delivery. With a median average score of 2.36 , it also marked a standard deviation of 1.116 . 
$\mathbf{V}_{25}$ : We have some techniques to reinforce our employees' sense of responsibility. $80.4 \%$ of the managers use these techniques to increase their employees' sense of responsibility. The median score is 2.84 (standard deviation 0.964).

\subsubsection{Obstacles to the Dimension of Integrity (Means V21, V23 and V25)}

The variables included address the managerial objectives in respecting organizational values and principles while executing the assigned tasks. Looking at the mean score of variables v21, v23 and v24, overall, $45.5 \%$ (100\%-54.5\%) of managers follow the dimension of integrity in the pursuit of their executive objectives.

After the presentation of the data from our 25 variables of the conceptual framework, it is necessary to employ the PCA (Principal Component Analysis) using SPSS analysis tools, in order to get a complete perspective of the five dimensions. The raw scores were standardized to allow for a uniform unbiased distribution of all variables. The correlation matrix was derived and the eigenvalues of these variables from the matrix were used for multivariate principal component extractions and the eigenvalues plotted on a screen plot graph as shown in Figure 1.

The first four significant principal components with a cut-off of $>1.0$ were selected. From the results we see that more than $63.87 \%$ of the total variance was attributed to these four principal components. The Varimax rotation with Kaiser Normalization was used to obtain a simple obstacle model. Table 3 shows the rotated component matrix of how each dimension variable loads onto each component. For our study, a factor loading cut-off of $>0.5$ was used. As seen in Table 3 that gives the KMO and Bartlett's test of sphericity results on the reliability of the data set, the KMO value of 0.926 is closer to 1.0 and thus statistically very significant. This adds good confidence and weighting to our PCA analysis.

The total variance explained by these 4 components was $63.879 \%$ (Table 4 ). The ranking of the dimensions, in order of importance were: the emotions dimension (getting a commitment to the objectives), the initiatives dimension (translating the objectives into projects), the rules dimension (clarifying and aligning the objectives) and finally the immediate actions dimension (value added actions and dealing with urgent matters).

\section{Findings}

Here we come to the crucial point of this article. The findings section will attempt to point the reader to the practical implications derived from the data analyses and their relation to the hypotheses we formulated. Practically, this section will demonstrate if our hypotheses were supported or not by the data and analyses. In order to do this we need to perform a PCA (Principal Component Analysis) and explain the total variance from the five components of the data analysis performed. We will start by explaining the five components from the PCA analysis beginning with the obstacles found in the analysis.

\subsection{Principal Component Analysis}

Principal Component Analysis "is a mathematical procedure that uses an orthogonal transformation to convert a set of observations of possibly correlated variables into a set of values of uncorrelated variables called principal components" (Jolliffe, 2002). It is a very useful tool in analyzing data and in making predictive models of what these data are showing. This is also the main reason why we use this analysis in our research. We want to assess if the data collected correlates to our hypotheses, what they are showing and what this can possibly mean for the future. We will discuss each component in detail and will identify what could hamper or improve managers' performance within the framework of their missions of public interest. We begin by exploring the data results from the drivers relating to the PCA analysis.

\section{Component 1: Drivers of the Emotions dimension}

The first component in our management / leadership obstacle model is a set of variables identified as follows: V6, V8, V7, V25, V9, V11, and V12 (Table 4). Most of the variables are related to the dimension of emotions (four out of seven emotions variables that form component 1). Indeed, this component consists of a set of variables that affect both the technical, cultural and emotional competencies when performing the execution of organizational strategies. It is also relevant to notice that after the rotation, some drivers that belonged to the Initiatives Driver and the Integrity Driver became, across the matrix transformation, linked to the Emotions Driver. This is the case for variables $\mathrm{V}_{11}, \mathrm{~V} 12$ and V25. The first two originally were part of the Dimension of Initiatives and V25 the Dimension of Integrity. In Table 4 the variables related to the Emotions Drivers are displayed in a descending order of importance. The higher loading of variables from the dimension of emotions marks the first component. This dimension addresses reflexive observations such as: problem recognition and the capacity to develop convictions and to get a commitment. With an eigenvalue of 5.65 , this component accounts for $37.70 \%$ of the total variance. V6, which is intended to observe the capacity of managers to 
encourage workers to adhere to their goals so that they are fully aware of their importance, accounts for the highest factor loading of 0.838 (Table 5).

\section{Component 2: Drivers from the Initiatives dimension}

This dimension is related to the variable drivers (Table 4) V12, V14, V15, V4 and V5. Here also it was noted that drivers V4 and V5, originally of the Dimension of Rules, came out of the matrix transformation and migrated to the Dimension of Initiatives. Table 4 displays the variables related to the initiatives drivers in a descending order of importance. Most of the variables are related to the size-taking initiatives ( 3 of the 5 initiatives variables that make up component 2). Indeed, this component 2 consists of a set of variables that affect only two major aspects such as rules and taking initiatives when executing organizational strategies. The second component was the Initiatives dimension. It was defined as an active capacity to experiment and select patterns to put new ideas into practice. The variance explained by this component was $10.49 \%$ with an eigenvalue of 1.57. In this cluster, $\mathrm{V}_{14}$ (utilizing various techniques of self-resolution at the employee levels in order to solve the management dilemmas they meet) had the highest factor loading of 0.763 (Table 5).

\section{Component 3: Drivers from the Rules dimension}

The drivers of these variables (Table 4) are $\mathrm{V}_{1}, \mathrm{~V}_{2}$, and $\mathrm{V}_{20}$. It is interesting to note that after the rotation matrix, these three main variables are the most critical. Also, after the rotation, the $V_{20}$ drivers that had been part of the immediate actions drivers came across the matrix transformation as drivers linked to rules dimension. This variable V20 has a loading factor of 0.63 . The third component characterizes the rules dimension and deals with clarifying and aligning the objectives. Unlike our conceptual framework, the dimension of rules seems less important with a low eigenvalue of 1.280 and accounts for $8.53 \%$ of the total variance. $\mathrm{V}_{1}$ (I have some techniques to clarify the expectations of our managers) has the highest factor loading of 0.828 . It is followed by $\mathrm{V}_{2}$ which has a factor loading of 0.789 in this component cluster which accounts for $8.53 \%$ of the variance (Table 5).

\section{Component 4: Drivers from the Immediate Actions dimension}

This concerns the variable drivers (Table 2) $\mathrm{V}_{20}$ and V17. The fourth component in our management/leadership performance model is the immediate actions dimension. This is defined as taking immediate actions to respond to urgent matters or to take value added actions. With the lowest eigenvalues of 1.071, the total variance explained by this component is $7.14 \%$. $\mathrm{V}_{20}$ (We dedicate a maximum of one

day each week to respond to urgent requests) has the highest factor loading of 0.817 . It is closely followed by V17 with a loading factor of 0.785 in a component which accounts for $6.743 \%$ of the total variance (Table 5).

Table 4. Rotated component matrix

\begin{tabular}{|c|c|c|c|c|}
\hline \multirow{2}{*}{ Drivers } & \multicolumn{4}{|c|}{ Components } \\
\hline & 1 & 2 & 3 & 4 \\
\hline V6 & .838 & & & \\
\hline V8 & .801 & & & \\
\hline V7 & .724 & & & \\
\hline V25 & .700 & & & \\
\hline V9 & .628 & & & \\
\hline V11 & .614 & & & \\
\hline V12 & .577 & .433 & & \\
\hline V14 & & .763 & & \\
\hline V15 & & .737 & & \\
\hline V4 & & .682 & & \\
\hline V5 & & .556 & & \\
\hline V1 & & & .828 & \\
\hline V2 & & & .789 & \\
\hline V20 & & & .630 & .817 \\
\hline V17 & & & & .785 \\
\hline
\end{tabular}

Extraction method: principal component analysis 


\subsection{Variance explained}

This second section analyses the total variance explained by our findings. A variance is used in probability to measure "how far a set of numbers is spread out from each other, describing how far the numbers lie from the mean (expected value)" (Goodman, 1960, p. 709). In our case, the closer they are to the components, the more chances there are to confirm our hypotheses. Below, in the table, we find a presentation of the factors drawn from each of the five dimensions of drivers encountered in the research.

Table 5. Total variance explained

\begin{tabular}{|c|c|c|c|c|c|c|c|c|c|}
\hline \multirow[t]{2}{*}{ Component } & \multicolumn{3}{|c|}{ Initial Eigenvalues } & \multicolumn{3}{|c|}{$\begin{array}{l}\text { Extraction Sums of Squared } \\
\text { Loadings }\end{array}$} & \multicolumn{3}{|c|}{$\begin{array}{c}\text { Rotation Sums of Squared } \\
\text { Loadings }\end{array}$} \\
\hline & Total & $\begin{array}{c}\% \text { of } \\
\text { Variance }\end{array}$ & Cumulative \% & Total & $\begin{array}{c}\% \text { of } \\
\text { Variance }\end{array}$ & Cumulative $\%$ & Total & $\begin{array}{c}\% \text { of } \\
\text { Variance }\end{array}$ & Cumulative \% \\
\hline 1 & 5.656 & 37.706 & 37.706 & 5.656 & 37.706 & 37.706 & 3.784 & 25.228 & 25.228 \\
\hline 2 & 1.574 & 10.494 & 48.200 & 1.574 & 10.494 & 48.200 & 2.425 & 16.169 & 41.396 \\
\hline 3 & 1.280 & 8.537 & 56.737 & 1.280 & 8.537 & 56.737 & 1.878 & 12.523 & 53.919 \\
\hline 4 & 1.071 & 7.142 & 63.879 & 1.071 & 7.142 & 63.879 & 1.494 & 9.960 & 63.879 \\
\hline
\end{tabular}

\section{Discussion}

\subsection{Overview of the Discussion}

This section is divided into two parts: the general and the specific. The general part explains more on the contribution of the observations drawn from the literature review. The specific part examines and discusses our five hypotheses formulated earlier. With respect to the findings on the subject of exploring the obstacles faced by public managers while executing their objectives, we intend to examine to what extent each of our hypotheses was supported. The results of the empirical analyses provided answers to our research questions.

The most significant finding stemming from this research is that, out of the five dimensions of the strategy execution skills used in the study, four dimensions, namely emotions, initiatives, rules and immediate actions, in this order of importance, were considered as essential by the respondents for their strategic skills. Though it is a generic finding, the implications of the results for both research and practice are really evident.

\subsection{General Part}

Our analysis brings to light the complementary perspective of four dimensions of strategy execution practices for the public managers. Both the analysis of the data and subsequent findings from the previous works highlight how strategy execution gathers multidimensional practices with varying complementary facets. As Waldo (1953) pointed out, for many decades administrators/managers have tended to use multiple variable solutions during the implementation of their strategies.

From our study and its correlations with the literature review, it resulted that these practices mainly deal with the aforediscussed dimensions of strategy execution. These dimensions include: dimension of emotions dealing with emotions and the capacity to get objectives; dimension of rules that focuses on commitment, objective clarification and alignment; dimension of initiatives which involves the translation of objectives into concrete projects; dimension of integrity which involves respecting the integrity of the values and principles; and the dimension of immediate actions with its proactive role to solve urgent matters. This was found to be consistent with the previous works in management and with the Kolb model (1984) and subsequent work (Richard \& Sabourin, 2009; Sabourin, 2009; Ayandé \& Sabourin, 2011).

\section{Component 1: Factors of the Emotions dimension}

This dimension of emotions includes the commitment of public managers towards the organization and work, how engaged they are with respect to the organization, their motivation or morale level, the extent to which they realize the importance of organizational goals and targets, and the extent to which they understand the sense of urgency in attaining the targets. Also, it covers the perception of how fairly they are treated in the organization and the conviction they apply to their work. Christensen and Laegreid (2008) observe that the heart, mind and hands of the public managers have to be connected to the work through the use of strategic skills. Hence, if the right attitude exists, capabilities can be developed (Stivens, 2008). 


\section{Component 2: Factors of the Initiatives dimension}

This dimension focuses on the translation of objectives into concrete projects and the continuous improvement of existing activities. It also includes the application of innovative ideas and experimentation for the betterment of the existing system. Bringing necessary changes and experimentation will encourage improvements within the organization.

\section{Component 3: Factors of the Rules dimension}

Rules are the guidelines or the path lines for achieving the objectives. The dream or vision of the organization has to be converted into specific objectives and targets which are concrete and tangible (Berry, 1994). This target has to be communicated clearly to the public managers and they must be precisely aware of what is expected from them. Defining the best attainable outcomes is the base of any strategic skills and by gaining clarity of the outcomes the organization will have a higher probability of aligning its investments to execute strategy (Fernandez and Rainey, 2006).

\section{Component 4: Factors of the immediate actions dimension}

This focuses on situations with uncertainties and the completion of tasks with a time tag fixed to them, while executing the strategies. Without adequate contingency plans and given the many uncertainties in the environment, completion of the execution within the time line becomes very difficult (Lane, 2000).

Summary of the discussion components

The dimension of emotions is considered as the foremost performance dimension when applying strategic skills. In other words, motivated and engaged public managers contribute to the successful execution of the strategy. The findings related to the dimension of initiatives can be applied to identify training and development needs, in order to fill the competency gap. Conversion of goals into concrete projects, techniques used for team-based management, techniques used for the self-resolution of managerial dilemmas all require a set of unique competencies. The findings related to the dimension of rules also have managerial implications. This dimension focuses on the clarity of communicating the expectations, systems to evaluate the results and supportive parameters and the process used for regular reviews, and it calls for precise identification, design and implementation of communication systems, evaluation systems and monitoring systems, respectively. Hence, management should strive to design perfect systems to ensure that the dimension of rules is followed. Though not all strategic skills have deadlines and contingencies, preparing for a crisis and planning for same will also ensure the support of the dimension of immediate actions. Since the dimension of integrity as a performance dimension is not considered, management should consider the reason for this.

\subsubsection{Specific Part}

In the above general discussion section we dealt with the general purpose of the research on the complementary perspective of the strategy execution practices implemented by managers. The specific scope of the research was to prove whether the hypotheses presented at the beginning of the paper are supported or not by the data gathered from the survey and their subsequent analyses. It is time to evaluate each of the hypotheses, one by one, and see if they have proved to be true or false.

H1: The management practices related to the dimension of emotions such as the resistance to change and the lack of commitment would be used more intensively by managers when executing their objectives than the other dimensions.

Our main hypothesis was supported. In accordance with the literature, our research highlights that practices that relate to the dimension of emotions (resistance to change and commitment from employees) appear to be the most critical dimension faced by managers in the execution of their strategy.

Our data suggest that the dimension of emotions is the most critical practice to 'change resistance' and explains $37.4 \%$ of the variance. The immediate actions dimension driver in our findings plays a secondary role as a management strategy execution practice. This is in contrast with some existing studies on strategy execution which have put a lot of emphasis on action-oriented practices and on the capacity to provide quick feedback (immediate action) from employees to explain the practices faced by managers.

The management practices that related to the dimension of emotions such as the capacity to develop convictions and to get a commitment represent a significant component of practices for managers when executing their objectives. Our hypothesis was thus empirically verified.

H2: The management practices that relate to the dimension of taking initiatives such as learning by doing and giving employees a sense of responsibility is a significant component of practices for managers when executing 
their objectives. Our hypothesis was supported. In line with the literature review, public managers are entrusted not with the responsibility of attaining the highest possible status, but with the responsibility to ensure that junior employees are given avenues which will allow them to learn. This learning involves attaining competent managerial practices and skills. Organizational success cannot be realized if management remains the boss and employees become enslaved. All levels should somehow be involved as each has a role to play in management. If duties are shared and distributed out, then it follows that organizational objectives will be achieved.

H3: The management practices that relate to the dimension of rules such as clarifying and aligning the objectives are a significant component of practices for managers when executing their objectives. Our hypothesis was supported.

Managers understand that a proper clarification of what should be done and how it should be achieved is essential in achieving maximum efficiency. A further step in clarifying organizational objectives will have public managers be ethical in their leadership and management roles. Several authors have highlighted some essential managerial disciplines regarding the formation of concepts and generalizations which integrate the observations and reflections (Sabourin, 2009; Richard \& Sabourin, 2009; Ayandé \& Sabourin, 2011). When formulating organizational rules it is imperative that these managers be ethical; they should therefore not formulate dictatorial rules which do not allow for managerial development, growth and learning.

Our data findings show that the dimension of rules takes up almost $56 \%$ of the cumulative variance, meaning that rules play a critical role in the management and objective achievement of public managers. This was found to be in line with the prior research, as shown in the literature review.

H4: The management practices that relate to the dimension of immediate actions, such as quick feedback and direct contacts, represent a significant component of practices for managers when executing their objectives. Our hypothesis was supported.

Managers do take advantage of the windows of opportunities to have direct contact with their employees, gather feedback from them and try to boost their morale. They found this to be a positive tool when implementing strategy.

H5: The management practices that relate to the dimension of integrity, such as practices related to value clarification, represent a significant component of practices for managers when executing their objectives. Our hypothesis was not supported. In the literature review, it was established that managers ought to be integral and whole in their management practices. Public managers should not withhold or falsify facts with the sole intention of achieving personal advancement or reward. It is important to note that the lack of adequate measures for the implementation of programs to promote integrity in the workplace can seriously hinder the ability of a manager to flatten the organizational obstacles. Our data show a $7.1 \%$ variance in integrity. This could support the hypotheses because public managers failed to clarify their objectives to the bottom-line employees. For public managers to achieve the highest verifiable success, they have to be ethical and disciplined in the performance of their work.

It seems that managers are so eager to implement strategy and spearhead change that many times they fail to clarify to their down-the-line employees the borders within which actions should be confined. This can either be justified with the sense of urgency they have or their sense that a radical change needs to take place in order to get the strategy executed. Another possible explanation is that, by challenging the company's guiding principles, they wish to make a radical change in the organizational culture, in order to make it easier for future strategy implementations. This way, "change" will be a consistent part of the organizational culture, weakening resistance to future objectives.

\subsection{Research Limitations/Values/Implications}

Our study contributes to the existing literature on strategy execution of management practices by examining, for the first time, the relative importance of specific practices in strategy execution in public management. Moreover, data from many other sectors will have to be collected to improve the external validity of our findings. Thus, future research will also have to be completed from other regions and from more sectors for a generalization of the results. Moreover, country specific differences need to be captured in the analysis.

On a more specific basis, this research demonstrated that the management practices that relate to the dimension of emotions $\left(\mathrm{H}_{2}\right)$, such as the capacity to develop convictions and to get a commitment, represent a significant component of practices for managers when executing their objectives, was supported. 
Also, $\mathrm{H}_{3}$ : The management practices that relate to the dimension of taking initiatives such as learning by doing and giving employees a sense of responsibility is a significant component of practices for managers when executing their objectives, was supported by the data gathered.

Also, $\mathrm{H}_{4}$ : The hypothesis within which the management practices that relate to the dimension of rules such as clarifying and aligning the objectives is a significant component of practices for managers when executing their objectives, was supported by the data gathered.

Also, $\mathrm{H}_{5}$ : The management practices that relate to the dimension of immediate actions such as quick feedback and direct contacts represent a significant component of practices for managers when executing their objectives, was supported by the data gathered.

One major remark / limitation of this research is that $\mathrm{H}_{6}$ : The hypothesis that the management practices that relate to the dimension of integrity such as practices related to value clarification, represent a significant component of practices for managers when executing their objectives, was not supported as a hypothesis by the data gathered.

\section{Conclusion}

This article explored the concept of strategy execution of management practices in public services. It highlights four components that play a key role in the execution of the objectives of managers. The main contribution of our investigation is to put into perspective the critical role played by emotions in the strategy execution practices of managers. The dimension of emotions deals with the capacity to get a commitment to the objectives of the organization. However, we found three other dimensions which are, in order of importance: the capacity to translate objectives into concrete projects (dimension of initiatives); the capacity to reinforce organizational values (dimension of integrity); the capacity to clarify objectives within the hierarchy and throughout the manager's department (dimension of rules). In contrast with our conceptual framework, we found that the fifth dimension (dimension of immediate actions) was split into two factors of immediate actions: urgent matters and valued-added actions.

In fact, and according to the conceptual framework of this study, we assert that the five dimensions are tools which public managers use to conduct their daily business. A balanced use of these five dimensions can imply a set of skills that are technical, social and contextually specific to the particular environment in which managers operate. However, it should be noted that the practice of these five dimensions by the manager can sometimes be conscious and / or unconscious, depending on his level of confrontation with the necessary achievement of strategic organizational objectives.

The study also investigates the importance played by dimensions such as emotions and resistance to change. The dimension of rules is a strategy based on a factual and empirical analysis of the situation in order to set up a coherent and rational system of managing the organization. It leads to a business model based on an analysis of facts and on their rational analysis. Regarding the role played by emotions, Frant (1993) argued that resistance to change represents a key factor in strategy execution and it should also take into account any resistance from managers. Some professionals and academics argue that the execution of strategy in an organization should be based on decentralized initiatives of individual innovation and small-scale improvement (Berry, 1994; Christensen and Laegreid, 2008; Altshuler and Behn, 1997). Thus, managers should rely on a constant experimentation of initiatives and improvement of existing activities (Sabourin, 2009; Ayandé \& Sabourin, 2011). This is labeled as the dimension of initiatives. But in the course of their daily routine, managers oftentimes encounter opportunities for fast decision making without the need for a prior established strategy. This is the dimension of immediate actions. Here the interaction with others is favoured only as far as it gives quick results; otherwise, managers have to exert leadership and decide fast. Their capacity to act fast with concrete actions in response to the situation, defines the success or failure of their organization (Frant, 1993). Authors like Christensen and Laegreid (2008) have argued that the value of transparency is important for the success of the implementation of a strategy and increase in organizational performance. Other values play an important role as well. That is why project managers try to transform team characteristics into a set of virtues (Altshuler and Behn, 1997). This is what they label an organization's dimension of integrity. Finally, the dimension of emotions is related to the development of convictions and commitment from employees. It deals with ways managers find and implement to clarify problems, reconcile divergent viewpoints and increase commitment from their employees. It is a way to motive and implement change and increase performance in strategy execution. To conclude, we reiterate that the dimension of emotions plays a significant role in the achievement of certain targets by public service managers. Moreover, our study reports the relevance of this dimension over the others. 


\section{References}

Altshuler, A. A., \& Behn, R. D. (1997). Innovation in American government: challenges, opportunities and dilemma. Brookings Industry Press, Washington D. C.

Ayandé, A., \& Sabourin, V. (2011). How Managers execute their strategic objectives? An empirical survey of drivers and practices. Asian Journal of Business and Management Sciences. 1(4), 63-81.

Berry, F. S. (1994). Innovation in public management: The adoption of strategic planning. Public Administration Review, 4(55), 322-330. http://dx.doi.org/10.2307/977379

Christensen, T., \& Laegreid, P. (2008). The challenge of coordination in central government organizations: The Norwegian Case. Public Organization Review, 2(8), 97-116. http://dx.doi.org/10.1007/s11115-008-0058-3

D’Agostino, M., \& Levine, H. (2010). Women in Public Administration. Ontario: Jones \& Bartlett Learning.

Damare, B. (Spring 2010). Workplace Learning to Improve IT Project Management. The Public Manager Journal, 2(38).

Ferguson, R. Paulin., \& M. Bergeron, J. (2010). Customer sociability and the total service experience: Antecedents of positive word-of-mouth intentions. Journal of Service Management, 1(21), 25-44. http://dx.doi.org/10.1108/09564231011025100

Fernandez, S., \& Rainey, H. G. (2006). Managing successful organizational change in the public sector. Public Administration Review, 2(66), 168-176. http://dx.doi.org/10.1111/j.1540-6210.2006.00570.x

Frant, H. (1993). Rules and Governance in the public sector: The case of civil service. American Journal of Political Science, 4(37), 990-1007. http://dx.doi.org/10.2307/2111540

Goodman, Leo A. (1960). On the exact variance of products. Journal of the American Statistical Association, December 1960, 708-713. http://dx.doi.org/10.1080/01621459.1960.10483369

Henisz, W. J. (1999). The institution and governance of economic reform: Theoretical extensions and applications. Public Management Review, 3(1), 349-371. http://dx.doi.org/10.1080/147190399000000011

Jolliffe I.T. (2002). Principal Component Analysis. Series: Springer Series in Statistics (2nd ed.) Springer, NY, 2002, XXIX, 487p28.

Jorgensen, T. B. (2006). Public values, their nature, stability and change: the case of Denmark. Public Administration Quarterly, 4(30), 365-398.

Karakosta, Ch. Doukas, H., \& Psarras, J. (2009). Shaping sustainable development strategies in Chile. International Journal of Climate Change and Management, 4(1). 382-399.

Kettl, D. F. (2002). The transformation of governance: public administration for twenty-first century America. JHU Press, Baltimore, MA.

Khan, A. (2008). Introduction to Public Administration. Lanham, MY: University Press of America.

Kolb, David A. \& Richard E. Boyatzis. (1995). From learning styles to learning skills: The executive skills profile . Journal of Managerial Psychology. Bradford: 1995. 10, Numéro 5; p. 3.

Kolb, David A. (1984). Experiential Learning: Experience as the Source of Learning and Development. Englewood Cliffs, New Jersey: Prentice-Hall.

Lane, E. (2000). The public sector: concepts, models and approaches. New York: SAGE Publications.

McLoughlin, D. (1985). A framework for integrated emergency management. Public Administration Review, 45, 165-172. http://dx.doi.org/10.2307/3135011

Metcalfe, L. (1993). Public Management: From imitation to innovation. Australian Journal of Public Administration, 3(54), 292-304. http://dx.doi.org/10.1111/j.1467-8500.1993.tb00281.x

Park, S. M., \& Rainey, H. G. (2008). Leadership and public service motivation in U.S. Federal Agencies. International Public Management Journal, 1(11), 109-142. http://dx.doi.org/10.1080/10967490801887954

Rainey, H. G., Backoff, R. W., \& Levine, C. H., (1976). Comparing Public and Private Organizations. Public Administration Review, 2(36), 233-244. http://dx.doi.org/10.2307/975145

Richard, É., \& Sabourin, V. (2009). Leadership experiential dans les services: une évaluation empirique de dimensions experiential. A Working paper Strategy Execution Research Group. 
Ring, P. S., \& Perry, J. L. (1985). Strategic Management in Public and Private Organizations: Implications of Distinctive Contexts and Constraints. The Academy of Management Review, 2(10), 276-286.

Sabourin, V. (2009). Experiential leadership: five strategies to improve the efficiency of services and transform organizations. Colloquium proceedings of (AIMS) Association Internationale de management stratégique.

Stivens, C. (Jan/Feb., 2008). The significance of the Administrative State. Public Administration Review, 53-56. http://dx.doi.org/10.1111/j.1540-6210.2007.00835.x

Stivers, C. (1994). The listening bureaucrat: Responsiveness in public administration. Public Administration Review, 4(54), 364-369. Retrieved from http://www.jstor.org/stable/977384 http://dx.doi.org/10.2307/977384

Van der wal, Z, Huberts, L, Van den hovel, H., \& Kolthoff, E. (2006). Central values of Government and business: Differences, Similarities and Conflicts. Public Administration Quarterly, 3(30).

Waldo, D. (1953). Ideas and Issues in Public Administration. New York: McGraw-Hill.

Warwick, D. P. (1975). A theory of Public Bureaucracy. Harvard university press, Cambridge, MA.

Zerbe, W. Hartel, Ch., \& Sshkanasy, N. [editors] (2010). Emotions and Organizational Behavior. London: Emerald Publishing. 